\title{
Amygdala Metabotropic Glutamate Receptor 1 Influences Synaptic Transmission To Participate In Fentanyl-Induced Hyperalgesia In Rats
}

\section{Tianyu Bai}

South-Central University for Nationalities https://orcid.org/0000-0002-9598-8191

Hengling Chen

South-Central University for Nationalities https://orcid.org/0000-0001-8129-2188

\section{Wenwu Hu}

South-Central University for Nationalities https://orcid.org/0000-0003-1028-0846

Jingtao Liu

South-Central University for Nationalities https://orcid.org/0000-0003-1569-4730

Xianguang Lin

South-Central University for Nationalities https://orcid.org/0000-0002-4875-0877

\section{Su Chen}

South-Central University for Nationalities https://orcid.org/0000-0001-7530-8198

\section{Fang Luo}

Huazhong University of Science and Technology https://orcid.org/0000-0003-4103-5712

\section{Xiaofei Yang}

South-Central University for Nationalities https://orcid.org/0000-0001-8383-2432

Jun Chen

Renmin Hospital of Wuhan University https://orcid.org/0000-0002-9312-4306

Chenhong Li ( $\sim$ lichen@mail.scuec.edu.cn )

South-Central University for Nationalities https://orcid.org/0000-0001-6234-6048

\section{Short Report}

Keywords: Pain, opioid-induced hyperalgesia, mGluR1, amygdala, CeLC

Posted Date: January 5th, 2022

DOI: https://doi.org/10.21203/rs.3.rs-1105473/v1

License: (c) (1) This work is licensed under a Creative Commons Attribution 4.0 International License.

Read Full License 


\section{Abstract}

The underlying mechanisms of opioid-induced hyperalgesia $(\mathrm{OIH})$ remain unclear. Herein, we found that the protein expression of metabotropic glutamate receptor 1 (mGluR1) was significantly increased in the right, but not in the left laterocapsular division of central nucleus of the amygdala (CeLC) in OIH rats. In CeLC neurons, the frequency and the amplitude of mini-excitatory postsynaptic currents (mEPSCs) were significantly increased in fentanyl group which were decreased by acute application of a mGluR1 antagonist, A841720. Finally, the behavioral hypersensitivity could be reversed by A841720 microinjection into the right CeLC. These results show that the right CeLC mGluR1 is an important factor associated with $\mathrm{OIH}$ that enhances synaptic transmission and could be a potential drug target to alleviate fentanylinduced hyperalgesia.

\section{Introduction}

Opioids produce strong analgesia and are mainly used to treat acute and chronic pain. Paradoxically, the administration of opioids may elicit a lower threshold of pain, which is defined as opioid-induced hyperalgesia (OlH) (Celerier et al. 2000; Mao 2002; Vanderah et al. 2001; Lee and Yeomans 2014). Prolonged opioid exposure also produces analgesic tolerance characterized as opioid tolerance, which has the same clinical manifestations as OlH. However, opioid tolerance can usually be suppressed by increasing the dosage of the opioid, whereas it may exacerbate $\mathrm{OIH}$. This contradictory phenomenon has led to the clinical use of opioids as a dilemma(Kim et al. 2014). As fentanyl, a potent synthetic opioid, and its derivatives are the most commonly used in clinical anesthesia and analgesia, more scientific research providing deeper insight into the mechanisms of $\mathrm{OlH}$ is needed.

Previous studies have implicated a prominent role for the laterocapsular division of the central amygdala (CeLC) in OlH development (Kissiwaa et al. 2019). Only elderly mice, not middle-aged or young mice, show elevated ERK1phoshhorylation in the right CeA following peripheral damage. ERK1 activation may account for age-related variations in pain sensitivity(Sadler et al. 2017). Our team and our collaborators discovered that extracellular signal-regulated kinase (ERK) activation in CeLC may contribute to OIH development by strengthening synaptic communication between CeLC neurons ( $\mathrm{Li}$ et al. 2017b). Calcium/calmodulin dependent protein kinase II alpha(CaMKIla) activity in the CeLC is also involved in $\mathrm{OIH}$ development ( $\mathrm{Li}$ et al. 2016). We are still working on this field to reveal the potential integrate pathways to regulate signal transmission in CelC during $\mathrm{OlH}$ occurs.

Several possible molecular mechanisms of OlH include sensitization of the central glutamatergic system, disruption of $\mathrm{Cl}^{-}$homeostasis, and changes in opioid receptors (Lee and Yeomans 2014; Roeckel et al. 2016). It has been recently discovered that the activation of the N-Methyl-d-aspartate (NMDA) receptor is intimately linked to $\mathrm{OlH}$. The cross-regulation between the ionotropic glutamate receptor $\mathrm{N}(\mathrm{GluN})$ and the mu-opioid receptor contribute to the transmission and modulation of nociceptive signals, which result in the development of OIH (Sanchez-Blazquez et al. 2013). Most studies on glutamatergic pathways in the $\mathrm{OIH} \mathrm{field} \mathrm{focused} \mathrm{on} \mathrm{the} \mathrm{ionotropic} \mathrm{glutamate} \mathrm{receptor(iGluR),} \mathrm{while} \mathrm{studies} \mathrm{on} \mathrm{the} \mathrm{central} \mathrm{metabotropic}$ 
glutamate receptor (mGluR) are rarely reported. Knockdown of spinal mGluR1 significantly diminishes cold hyperalgesia, heat hyperalgesia, and mechanical allodynia by restoring morphine sensitivity and reducing NMDA sensitivity in neuropathic rats (Fundytus et al. 2001). Further research is necessary to confirm the particular involvement of mGluR in $\mathrm{OlH}$.

Here, we describe a hyperalgesia model prepared by repeated injection of fentanyl in rats. Notably, increased mGluR1 expression in CeLC was detected in OIH rats. The frequency and amplitude of mEPSCs in CeLC neurons were also increased during $\mathrm{OIH}$ and then recovered to the normal level by blocking mGluR1 in brain slices. Mechanical and heat sensitivities were recovered by treatment with an mGluR1 receptor antagonist in the right CeLC of the OlH model. We focused on the modulation of CeLC mGluR1 in fentanyl-stimulated hyperalgesia.

\section{Materials And Methods}

\subsection{Animals}

Male adolescent Sprague-Dawley rats weighing 90 to $110 \mathrm{~g}$ were obtained from Hubei Research Center of Laboratory Animals. The rats were housed in cages under standard laboratory conditions of temperature $\left(20-22^{\circ} \mathrm{C}\right)$ and relative humidity (55-65\%) with a 12-h light/dark cycle. All rats were provided with food and water available ad libitum. Animal handling was approved by the Animal Care and Use Committee of South-Central University for Nationalities(2019-SCUEC-AEC-022) and conformed to the guidelines of the International Association for the Study of Pain (Zimmermann 1983). The rats were randomly grouped and the experimenters were blinded to group assignments and data analysis.

\subsection{Behavioral assessments}

The fentanyl citrate solution (Yichang Humanwell Pharmaceutical Co. Ltd., Yi Chang, Hubei, China) was administered as four subcutaneous bolus injections at 15-min intervals using a $60 \mu \mathrm{g} / \mathrm{kg}$ dose per injection (fentanyl citrate concentration $50 \mu \mathrm{g} / \mathrm{ml}, 120 \mu \mathrm{l} / 100 \mathrm{~g}, 4$ times) (Celerier et al. 2000). The same volume of $0.9 \%$ saline was administered to the vehicle group. Mechanical and heat sensitivities were measured before injection as the baseline data. Mechanical allodynia was tested by measuring the withdrawal threshold for a series of von Frey filaments (beginning with $1.0 \mathrm{~g}$; North Coast, San Jose, CA, USA). The thermal pain threshold was tested using a model BME-410C radiant thermal stimulator (Biomedical Engineering, Boerni Science and Technology Co. Ltd., Guangzhou, China).

\subsection{Western blot and immunohistochemistry}

Brain slices (1 mm, brain matrix 0-175 g) were prepared using a model BJK-001 device (J\&K Seiko, DongGuan, China). CeLCs were collected using a patching technique guided by a dissecting microscope following the Allen Brain atlas (Zapala et al. 2005; Han et al. 2005). Then 6 CeLCs per one sample were lysed using radio-immunoprecipitation assay (RIPA) buffer and protein concentrations were determined using the Coomassie (Bradford) assay kit (23200; Pierce Biotechnology, Rockford, IL, USA). Samples containing equal amounts of protein were separated using 10\% SDS-PAGE and blotted onto 
polyvinylidene fluoride membranes, blocked with $5 \%(\mathrm{w} / \mathrm{v})$ bovine serum albumin in Tris-buffered salineTween (TBST) at room temperature for $1 \mathrm{~h}$, and then incubated with the primary antibody at $4^{\circ} \mathrm{C}$ overnight. The antibody was anti-glyceraldehyde 3-phosphate dehydrogenase (GAPDH, 1:500 dilution; Boster, Wuhan, China) or anti-mGluR1 (1:2000 dilution, cat\#07-617, lot \#2207272; MilliporeSigma)(Shin et al. 2015). After several washes, the blots were incubated with the secondary antibody (1:10000 dilution; Boster). The immunoreactive bands corresponding to mGluR1 were analyzed using the ChemiDoc system (Bio-Rad, Hercules, CA, USA).

For immunohistochemistry, $7 \mu$ m-thick CeLC sections were cut on a freezing sliding microtome and rinsed in anti-mGluR1 antibody (1:100) overnight at $4^{\circ} \mathrm{C}$. Detection was performed using a DAB Substrate kit (Boster) following the manufacturer's instructions. Images were taken using a digital camera (IX-81, Olympus Corp, Tokyo, Japan, magnification, ×400).

\subsection{Cannula construction and implantation}

The rats were intraperitoneally injected with $10 \%$ chloral hydrate $(3 \mathrm{~mL} / \mathrm{kg})$ and then positioned on a stereotaxic frame (Zenda, Austin, TX, USA). The right CeLC was determined according to the rat brain atlas (Paxinos and Watson 2006), and infusion cannulas (RWD Life Science, Shenzhen, China) were implanted into it. The final stereotaxic coordinates in young rats should be $2.2 \mathrm{~mm}$ caudal to Bregma, 4.2 $\mathrm{mm}$ lateral to midline, and $7.5 \mathrm{~mm}$ ventral to dura (Li et al. 2016; Li et al. 2017a; Butler et al. 2011). Each cannula was fitted with a dummy cannula (RWD Life Science) to reduce the incidence of occlusion. After cannulation, rats were housed individually and allowed to recover for 7 days.

The noncompetitive mGluR1 antagonist, A841720 (SML0818, MilliporeSigma, Darmstadt, Germany) was completely dissolved in dimethyl sulfoxide (DMSO) and then diluted to $20 \mathrm{nmol} / \mathrm{mL}$ with saline (ElKouhen et al. 2006; More et al. 2007). DMSO (0.1\%) was used as a vehicle control. A841720 was microinjected $6 \mathrm{~h}$ after fentanyl administration. Timeline diagrams were constructed for illustrative purposes (see the details in Fig. 3A, 4A, and 5A). An infusion volume of $0.3 \mu \mathrm{L}$ was pumped into the right CeLC over the course of $2 \mathrm{~min}$ at a rate of $0.15 \mu \mathrm{L} / \mathrm{min}$. The needle was kept in place for $2 \mathrm{~min}$ and then gently removed. Success of the injection in the CeLC was histologically verified at the conclusion of the procedures by injecting $0.3 \mu \mathrm{L}$ of eosin. The data were excluded if the cannula locations were outside the right CeLC.

\subsection{Measurement of CeLC glutamate levels}

The glutamate concentrations of right CeLC were determined separately $30 \mathrm{~min}$ after the microinjection of A841720 using an ELISA kit (RA21320; Bioswamp, Wuhan, China). Following the manual directions, each sample including seven CeLCs was transferred into a well, incubated with biotinylated detection antibody, and then labeled with horseradish peroxidase-conjugated (HRP) substrate. The optical density at $450 \mathrm{~nm}$ was recorded using a spectrophotometer (Varioskan LUX, Thermo Fisher Scientific, MA, USA). This value was proportional to the glutamate concentration. 


\subsection{Brain slice preparation and electrophysiological recording}

\subsubsection{Slice preparation}

The rats were prepared $6 \mathrm{~h}$ after the last injection of fentanyl and killed by decapitation. Brain tissue was sectioned into coronal slices containing the amygdala by a vibrating slicer (VT1000S, Leica Nussloch, Germany). The components of the dissection buffer (in $\mathrm{mM}$ ) were: 213 sucrose, $3 \mathrm{KCl}, 1 \mathrm{NaH}_{2} \mathrm{PO}_{4}, 0.5$ $\mathrm{CaCl}_{2}, 5 \mathrm{MgCl}_{2}, 26 \mathrm{NaHCO}_{3}$, and 10 glucose. The artificial cerebrospinal fluid (ACSF) contained (in mM): $125 \mathrm{NaCl}, 5 \mathrm{KCl}, 1.2 \mathrm{NaH}_{2} \mathrm{PO}_{4}, 2.6 \mathrm{CaCl}_{2}, 1.3 \mathrm{MgCl}_{2}, 26 \mathrm{NaHCO}_{3}$, and 10 glucose. Both solutions were equilibrated to a pH of 7.3 to 7.4 and oxygenated with a mixture of $95 \% \mathrm{O}_{2}$ and $5 \% \mathrm{CO}_{2}$. The right hemisphere coronal slices $(300 \mu \mathrm{m})$ containing CeLC were cut with a vibrating microtome in pre-cooled dissection buffer. The slices were incubated with ACSF at room temperature for $1 \mathrm{~h}$ and transferred to the recording chamber with a continuous perfusion of $2 \mathrm{~mL} / \mathrm{min}$ of ACSF. Only one neuron was recorded from each brain slice.

\subsubsection{Patch-clamp recordings}

The whole-cell voltage-clamp was used to record mEPSCs in the amygdala slices. The A841720 solution $(20 \mathrm{nmol} / \mathrm{mL})$ was added to brain slices $10 \mathrm{~min}$ after the baseline recording. The pipette $(4-6 \mathrm{M} \Omega)$ was made from borosilicate glass capillaries (WPI, Sarasota, FL, USA) filled with the inner solution, containing (in $\mathrm{mM}$ ): $145 \mathrm{KCl}, 5 \mathrm{NaCl}, 10 \mathrm{HEPES}, 5$ EGTA, $4 \mathrm{Mg}$-ATP, and $0.3 \mathrm{Na}_{3}$-GTP. Data were acquired using an EPC-10 amplifier (HEKA, Lambrecht, Germany) and PATCHMASTER software (HEKA), and analyzed using Clampfit (Molecular Devices, Sunnyvale, CA, USA). High ( $>2 G \Omega$ ) seal resistances and low $(<20 M \Omega)$ series resistances were checked throughout the experiment to ensure high-quality recordings. Picrotoxin $(50 \mu \mathrm{M})$ and tetrodotoxin $(1 \mu \mathrm{M})$ were used to block the gamma aminobutyric acid $A\left(G A B A_{A}\right)$ and sodium channels, respectively, during the recording of the mEPSCs at a holding potential of $-70 \mathrm{mV} 10$ min before and after drug administration. The Mini Analysis Program 6.0 (Synaptosoft Inc., Fort Lee, NJ, USA) and Clampfit software were used to analyze the frequency and amplitude of mEPSCs.

\subsection{Statistical analyses}

The effect of $\mathrm{A} 841720$ on the change in glutamate concentrations (Fig. 2C), frequency and amplitude of mEPSCs (Fig. 3C, D), and mechanical and heat sensitivities (Fig. 4B, C) were all analyzed by ordinary oneway ANOVA followed by Tukey test. Cumulative distributions of mEPSC inter-event interval and amplitude were analyzed with a Kolmogorov-Smirnov test. All statistical analyses and graphs were performed using GraphPad Prism 8.0 (GraphPad Software, San Diego, CA, USA). All data were presented as Mean \pm SD. Significant differences were defined as $P<0.05$. All statistical analyses were blinded with respect to treatment groups.

Sample size was estimated after data was collected base on partial eta squared observed in the experiments(Kadam and Bhalerao 2010). The minimal number of animals was determined to obtain a 
power of $80 \%$ at an alpha level of 0.05 . It was calculated with G*Power 3.1.

\section{Results}

\subsection{Fentanyl increased the expression of mGluR1 in CeLC}

In the previous study we used OIH models induced by Fentanyl administration (Li et al. 2017b; Li et al. 2016), and in this study we continued to use this model. OIH models were prepared by consequent injections of fentanyl. Compared with the control, fentanyl administration exhibited a significant increase initially (an acute analgesic effect), followed by a substantial decrease in the mechanical and thermal nociceptive threshold (hyperalgesia) (S. Fig. 1A, B). The mechanical or thermal pain threshold reached the lowest point 5 6 $\mathrm{h}$ after the last injection. The behavior hyperalgesia was reinstated to the normal level on the 5 th day (S. Fig. 1A, B). So sampling time point was set at $6 \mathrm{~h}$ after the last fentanyl administration.

The effect of fentanyl on mGluR1 protein expression was investigated using Western blot and immunohistochemistry. The mGluR1 level in the right CeLC was markedly improved as compared to the vehicle treatment. (Fig. 1B). The experiment was repeated independently in triplicate with similar results(S. Fig. 2). And the sections of right CeLC labeled with mGluR1 antibodies also displayed positive staining around the plasma membrane following fentanyl exposure (Fig. 1C).

\section{2 mGluR1 blockage by A841720 can suppress the upregulation of fentanyl-induced glutamate in CeLC}

The CeLC glutamate concentration was elevated in OIH rats but repressed after microinjection of the mGluR1 antagonist A841720 into the right CeLC (Fig. 2), suggesting that a reduced glutamate content acting on mGluR1 may contribute to the nodes within the ascending nociceptive pathways. In addition, A841720 can significantly down-regulate the glutamate content under the normal condition as well (Fig. 2).

\section{3 mGluR1 blockage can inhibit the increased frequency and amplitude of mEPSCs in CeLC slices induced by fentanyl injection}

Spontaneous mEPSCs can be used to characterize synaptic transmission changes responsible for inflammatory and neuropathic pain. mEPSCs were recorded at -70mV from CeLC neurons after fentanyl treatment in the presence or absence of the mGluR1 antagonist A841720. Normalized cumulative distribution analysis showed that A841720 caused an increase in the inter-event interval in fentanyl groups (Fig. 3). Compared to the control group, both the frequency and amplitude of mEPSCs of the right CeLC neurons in the fentanyl group were initially higher which were reversed after A841720 was directly applied to the brain slices $(20 \mathrm{nmol} / \mathrm{mL}$ in the recording chamber) $(* \star \star P<0.001)$ (Fig. 3). The recording of 
mGluR1-mediated mEPSCs, reflecting the release of neurotransmitters, revealed that CeLC mGluR1 is critical for the persistence of $\mathrm{OIH}$ by influencing synaptic transmission.

\subsection{Microinjection of A841720 to right CeLC alleviated fentanyl-induced hyperalgesia}

To further explore the role of CeLC mGluR1 in OIH, the mGluR1 blocker A841720 was microinjected into the right CeLC following fentanyl administration. Mechanical and heat sensitivity were significantly reduced 30 minutes after $A 841720$ microinjection compared with the vehicle-injected fentanyl group (Fig. 4). Inhibition of mGluR1 by A841720 in CeLC directly alleviated pain. These results implied that the activation of CeLC mGluR1 is critical for the development of hyperalgesia.

\section{Discussion}

Opioid administration is one of the most common methods of managing moderate to severe pain in patients of all ages. All opioids induce adverse effects that include opioid tolerance and/or hyperalgesia, which are characterized in relation to analgesic approach, sex, and age. Marked sex differences in the magnitude of opioid effects upon hyperalgesia have been described in humans and in animal models for reasons that remain unclear (Barrett et al. 2003; Holtman and Wala 2005; Bodnar and Kest 2010; Wasserman et al. 2015). NMDA receptor antagonists are shown to block hyperalgesia generated by highdose opioid exposure in male mice, while melanocortin-1 receptor antagonists are found to block hyperalgesia in female mice. (Juni et al. 2010). Prolactin inhibition prevented the occurrence of OIH in a female-selective manner (Chen et al. 2020). Interestingly, several male-specific genes have been implicated in the regulation of peripheral and spinal nociceptive processing during OIH (unpublished data, manuscript in preparation). For research continuity, our team attempted to delineate the molecular basis underlying male sensitivity to opioid antinociception. The aim was to better understand the distinct mechanisms of hyperalgesia between gender and clinical and therapeutic consequences.

The use of opioids has increased in adolescents undergoing surgical procedures (Kotzer 2000; Maxwell et al. 2005; Engelhardt et al. 2008; Tripi et al. 2015). Most relevant studies on OIH emphasized the need to explore the mechanistic basis exclusively in adult subjects. Instead, our research highlights the molecular underpinnings in adolescents. For this, rats at postnatal days 28-35 (P28-35) were used in all the experiments. The rostroventral medulla (RVM) of the brainstem has a powerful excitatory effect on spinal nociception from birth up to P21 in rats. Subsequently (P28 to adult), the influence of the RVM shifts to biphasic facilitation-inhibition (Hathway et al. 2009). The pain control system, which decreases the facilitation of spinal nociception rather than suppressing pain transmission in the preadolescent rat (P21), is mediated by mu-opioid receptor pathways in the RVM (Hathway et al. 2012). Naloxone, an opioid receptor antagonist, can prevent the most common opioid-induced side effects in children and adolescents being treated for acute postoperative pain (Maxwell et al. 2005). After pediatric scoliosis surgery, an intraoperative infusion of the NMDA receptor antagonist ketamine was reportedly unable to prevent the development of remifentanil-induced tolerance and decreased postoperative morphine 
consumption. Intraoperative infusion of the NMDA receptor antagonist ketamine reportedly could not prevent the development of remifentanil-induced tolerance and decreased postoperative morphine consumption after pediatric scoliosis surgery (Engelhardt et al. 2008; Tripi et al. 2015). The present finding demonstrates that the use of an mGluR1 antagonist in adolescents may increase the pain threshold during $\mathrm{OIH}$ in an animal model. Further studies will be informative to elucidate the clinical efficacy and significance of this approach.

The amygdala is located in the medial temporal lobe and is comprised of the lateral-basolateral complex (LA/BLA), intercalated cell mass (ITC), and central nucleus (CeA). The LA/BLA neurons receive polymodal sensory signals from the cortical and thalamic regions. ITC cells receive excitatory inputs from the medial prefrontal cortex (mPFC) (Thompson and Neugebauer 2017; Neugebauer et al. 2020). Nociceptive information can be delivered to the $\mathrm{CeA}$, which serves as the major output nucleus, and glutamatergic synaptic responses are involved in pain transmission (Tully et al. 2007; Neugebauer et al. 2004). CeLC, defined as the nociceptive amygdala, has been identified as a target of the spino-parabrachio-amygdaloid tract. The CeLC receives affect-related inputs from the LA/BLA and contributes critically to pain modulation via projections to the brainstem (Gauriau and Bernard 2002; Carrasquillo and Gereau 2007; Ji and Neugebauer 2008). Paradoxical hyperalgesia can be induced by the mu-opioid agonist endomorphin2 administered in the centromedial amygdala of rats (Terashvili et al. 2007). The CeLC may serve as a central site for the modulation of pain perception in the OlH process.

Interestingly, there is evidence to suggest pain-related hemispheric lateralization in the amygdala. There is no discernible difference in pain sensation in both the right and left amygdala under normal conditions, whereas a predominant involvement of the right amygdala has been observed in nociceptive responses to inflammatory and neuropathic pain stimuli (Thompson and Neugebauer 2017). An inhibitor of protein kinase A decreased the activity of right CeLC neurons after the induction of arthritis (Ji and Neugebauer 2009). ERK activation occurs in the right CeA and plays a dominant role in inflammation-induced peripheral hypersensitivity (Carrasquillo and Gereau 2008). Engagement of opioid receptor signaling in the right CeA contributes to pain responses produced by morphine (Nation et al. 2018; Navratilova et al. 2020). This agrees with the previous finding that the modulation of pain by mGluR1 activation is functionally lateralized to the right hemisphere.

Although the mechanism of $\mathrm{OlH}$ is unclear, it is conceivable that the activation of the glutamatergic system is involved. Glutamate is a major excitatory neurotransmitter in the mammalian nervous system. The compound is important in pain pathways, where it participates in transmitting nociceptive signals from the peripheral nociceptors to the central nervous system. There are two types of glutamate receptors: the ionotropic type (iGluRs) and the metabotropic type (mGluRs). To date, eight subtypes of mGluR have been identified in the nervous system. They include group I mGluR1/mGluR5, group II mGluR2/mGluR3, group III mGluR4, and mGluR6-8(Palazzo et al. 2019).

Group I mGluR-induced nociceptive processing in amygdala neurons participates in emotional-affective pain modulation through a mechanism that involves reactive oxygen species (Ji and Neugebauer 2010). 
There are the enhanced synaptic transmission at the nociceptive parabrachial $(\mathrm{PB}) \rightarrow \mathrm{CeA}$ synapse and the increased excitability which results in the altered output from the CeA in the arthritis model. The enhanced synaptic transmission and altered excitability in the CeA neurons were accompanied by the upregulation of mGluR1 and mGluR5(Neugebauer et al. 2003). Blocking mGluR1 rather than mGluR5 can reverse pain-related alterations in excitatory and inhibitory transmission in CeLC neurons from arthritic rats(Ren and Neugebauer 2010). Rescue of impaired endocannabinoid-dependent mGluR5 facilitation can restore mPFC output, which inhibits pain behaviors in arthritic rats (Kiritoshi et al. 2016). Activation of group II mGluR2 and mGluR3 subtypes can decrease neurotransmitter release in the synaptic cleft and inhibit spinal nociceptive processing in arthritis pain model (Mazzitelli and Neugebauer 2019; Mazzitelli et al. 2018). Recent evidence revealed that group III mGluR7 (pain enhancing) acts as a gatekeeper to regulate the flow of information to CeLC neurons under normal circumstances, but not during pain. Presynaptic mGluR8 (pain inhibiting) inhibits excitatory transmission in the CeLC in the state of pain (Ren et al. 2011). The data also suggest that mGluR8 and mGluR5 counteract the neuroprotective effect of ultra-micronized palmitoylethanolamide on long-term potentiation in conditions of neuropathic pain (Boccella et al. 2019).

In this study, the potential role of mGluR1 in the OIH process was explored. A proportion of the cellular glutamate pool was stored in the synaptic gap, and the protein expression of mGluR1 was increased after fentanyl exposure. We noticed that application of A841720 reduced glutamate content both under the normal condition and in $\mathrm{OIH}$, suggesting unknown influences on normal mGluR1-mediated function in the amygdala when mGluR1 antagonist was used in the treatment of $\mathrm{OlH}$. The potential side effects should be closely investigated. The increase and decrease in mGluR1 levels depended on the dynamic balance of its production and internalization. Presynaptic function was measured by mEPSC frequency and postsynaptic responsiveness was determined by mEPSC amplitude. CeLC cells displayed significantly increased mEPSC frequency and amplitude in the OIH process, which could be restored by blockade of mGluR1 using A841720. It's not common that mGluR1 blockade reduces both the frequency and amplitude of mEPSCs. There are some similar cases. The mEPSC frequency and amplitude recorded in anterior cingulate cortex neurons were obviously increased after peripheral nerve ligation (Xu et al. 2008). Tumor necrosis factor-alpha significantly increased mEPSC frequency and amplitude in the immature superficial dorsal horn neurons after the spared nerve injury (Li et al. 2009). Our findings indicated that CeLC mGluR1 may upregulate presynaptic neurotransmitter release and postsynaptic responsiveness as well to induce behavioral hyperalgesia.

The collective findings indicate the involvement of mGluR1 in the fentanyl-induced behavioral hyperalgesia, which may be related to the enhanced synaptic transmission in the right CeLC. Further prospective data are needed, which may help patients recover from $\mathrm{OlH}$.

\section{List Of Abbreviations}

Opioid-induced hyperalgesia (OIH); metabotropic glutamate receptor (mGluR); laterocapsular division of central nucleus of the amygdala (CeLC); mini-excitatory postsynaptic currents (mEPSCs); extracellular 
signal-regulated kinase (ERK); N-Methyl-d-aspartate(NMDA); ionotropic glutamate receptor N(GluN); ionotropic glutamate receptor(iGluR); artificial cerebrospinal fluid (ACSF); rostroventral medulla of the brainstem (RVM); lateral/basolateral amygdala nuclei (LA/BLA); intercalated cell mass of the amygdala (ITC); central nucleus of the amygdala (CeA); medial prefrontal cortex (mPFC).

\section{Declarations}

\section{Conflict of Interest}

The authors declare that the research was conducted in the absence of any commercial or financial relationships that could be construed as a potential conflict of interest.

\section{Author Contributions}

Chenhong Li, Hengling Chen, and Tianyu Bai designed the study; Hengling Chen, Tainyu Bai, Wenwu Hu and Jingtao Liu implemented the experiments; Hengling Chen Xianguang Lin, Su Chen, Fang Luo, and Xiaofei Yang analyzed data; Tianyu Bai, Hengling Chen and Chenhong Li drafted the paper. Tianyu Bai and Hengling Chen contributed equally to this work.

\section{Funding}

This work was supported by National Natural Science Foundation of China (grant nos. 31870771区 31500996ه3212018ه81271234, 81771196), National Science Foundation of Hubei (2020CFA025), Special Funds of Basic Research Operating Expenses for Universities of China (CZY17030, CZY18027》 CZY19023凶 CZQ20004), and Hubei Key Laboratory of Medical Information Analysis and Tumor Diagnosis \& Treatment (PJS140011610).

\section{Acknowledgments}

\section{Data Availability Statement}

The authors acknowledge that the data presented in this study must be deposited and made publicly available in an acceptable repository, prior to publication.

\section{References}

1. Barrett AC, Smith ES, Picker MJ (2003) Capsaicin-induced hyperalgesia and mu-opioid-induced antihyperalgesia in male and female Fischer 344 rats. J Pharmacol Exp Ther 307(1):237-245. doi:10.1124/jpet.103.054478

2. Boccella S, Marabese I, lannotta M, Belardo C, Neugebauer V, Mazzitelli M, Pieretti G, Maione S, Palazzo E (2019) Metabotropic Glutamate Receptor 5 and 8 Modulate the Ameliorative Effect of Ultramicronized Palmitoylethanolamide on Cognitive Decline Associated with Neuropathic Pain. Int J Mol Sci 20(7). doi:10.3390/ijms20071757 
3. Bodnar RJ, Kest B (2010) Sex differences in opioid analgesia, hyperalgesia, tolerance and withdrawal: central mechanisms of action and roles of gonadal hormones. Horm Behav 58(1):7281. doi:10.1016/j.yhbeh.2009.09.012

4. Butler RK, Nilsson-Todd L, Cleren C, Lena I, Garcia R, Finn DP (2011) Molecular and electrophysiological changes in the prefrontal cortex-amygdala-dorsal periaqueductal grey pathway during persistent pain state and fear-conditioned analgesia. Physiol Behav 104(5):1075-1081. doi:10.1016/j.physbeh.2011.05.028

5. Carrasquillo Y, Gereau RWt (2007) Activation of the extracellular signal-regulated kinase in the amygdala modulates pain perception. The Journal of neuroscience: the official journal of the Society for Neuroscience 27(7):1543-1551. doi:10.1523/JNEUROSCI.3536-06.2007

6. Carrasquillo Y, Gereau RWt (2008) Hemispheric lateralization of a molecular signal for pain modulation in the amygdala. Mol Pain 4:24. doi:10.1186/1744-8069-4-24

7. Celerier E, Rivat C, Jun Y, Laulin JP, Larcher A, Reynier P, Simonnet $G$ (2000) Long-lasting hyperalgesia induced by fentanyl in rats: preventive effect of ketamine. Anesthesiology 92(2):465-472. doi:10.1097/00000542-200002000-00029

8. Chen Y, Moutal A, Navratilova E, Kopruszinski C, Yue X, Ikegami M, Chow M, Kanazawa I, Bellampalli SS, Xie J, Patwardhan A, Rice K, Fields H, Akopian A, Neugebauer V, Dodick D, Khanna R, Porreca F (2020) The prolactin receptor long isoform regulates nociceptor sensitization and opioid-induced hyperalgesia selectively in females. Sci Transl Med 12(529). doi:10.1126/scitranslmed.aay 7550

9. El-Kouhen O, Lehto SG, Pan JB, Chang R, Baker SJ, Zhong C, Hollingsworth PR, Mikusa JP, Cronin EA, Chu KL, McGaraughty SP, Uchic ME, Miller LN, Rodell NM, Patel M, Bhatia P, Mezler M, Kolasa T, Zheng GZ, Fox GB, Stewart AO, Decker MW, Moreland RB, Brioni JD, Honore P (2006) Blockade of mGluR1 receptor results in analgesia and disruption of motor and cognitive performances: effects of A-841720, a novel non-competitive mGluR1 receptor antagonist. Br J Pharmacol 149(6):761-774. doi:10.1038/sj.bjp.0706877

10. Engelhardt T, Zaarour C, Naser B, Pehora C, de Ruiter J, Howard A, Crawford MW (2008) Intraoperative low-dose ketamine does not prevent a remifentanil-induced increase in morphine requirement after pediatric scoliosis surgery. Anesth Analg 107(4):1170-1175.

doi:10.1213/ane.0b013e318183919e

11. Fundytus ME, Yashpal K, Chabot JG, Osborne MG, Lefebvre CD, Dray A, Henry JL, Coderre TJ (2001) Knockdown of spinal metabotropic glutamate receptor $1(\mathrm{mGluR}(1))$ alleviates pain and restores opioid efficacy after nerve injury in rats. Br J Pharmacol 132(1):354-367.

doi:10.1038/sj.bjp.0703810

12. Gauriau C, Bernard JF (2002) Pain pathways and parabrachial circuits in the rat. Exp Physiol 87(2):251-258. doi:10.1113/eph8702357

13. Han JS, Li W, Neugebauer V (2005) Critical role of calcitonin gene-related peptide 1 receptors in the amygdala in synaptic plasticity and pain behavior. The Journal of neuroscience: the official journal of the Society for Neuroscience 25(46):10717-10728. doi:10.1523/JNEUROSCI.4112-05.2005 
14. Hathway GJ, Koch S, Low L, Fitzgerald M (2009) The changing balance of brainstem-spinal cord modulation of pain processing over the first weeks of rat postnatal life. J Physiol 587(Pt 12):29272935. doi:10.1113/jphysiol.2008.168013

15. Hathway GJ, Vega-Avelaira D, Fitzgerald M (2012) A critical period in the supraspinal control of pain: opioid-dependent changes in brainstem rostroventral medulla function in preadolescence. Pain 153(4):775-783. doi:10.1016/j.pain.2011.11.011

16. Holtman JR Jr, Wala EP (2005) Characterization of morphine-induced hyperalgesia in male and female rats. Pain 114(1-2):62-70. doi:10.1016/j.pain.2004.11.014

17. Ji G, Neugebauer V (2008) Pro- and anti-nociceptive effects of corticotropin-releasing factor (CRF) in central amygdala neurons are mediated through different receptors. J Neurophysiol 99(3):12011212. doi:10.1152/jn.01148.2007

18. Ji G, Neugebauer V (2009) Hemispheric lateralization of pain processing by amygdala neurons. J Neurophysiol 102(4):2253-2264. doi:10.1152/jn.00166.2009

19. Ji G, Neugebauer V (2010) Reactive oxygen species are involved in group I mGluR-mediated facilitation of nociceptive processing in amygdala neurons. J Neurophysiol 104(1):218-229. doi:10.1152/jn.00223.2010

20. Juni A, Cai M, Stankova M, Waxman AR, Arout C, Klein G, Dahan A, Hruby VJ, Mogil JS, Kest B (2010) Sex-specific mediation of opioid-induced hyperalgesia by the melanocortin-1 receptor. Anesthesiology 112(1):181-188. doi:10.1097/ALN.0b013e3181c53849

21. Kadam P, Bhalerao S (2010) Sample size calculation. International journal of Ayurveda research 1(1):55-57. doi:10.4103/0974-7788.59946

22. Kim SH, Stoicea N, Soghomonyan S, Bergese SD (2014) Intraoperative use of remifentanil and opioid induced hyperalgesia/acute opioid tolerance: systematic review. Front Pharmacol 5:108. doi:10.3389/fphar.2014.00108

23. Kiritoshi T, Ji G, Neugebauer V (2016) Rescue of Impaired mGluR5-Driven Endocannabinoid Signaling Restores Prefrontal Cortical Output to Inhibit Pain in Arthritic Rats. The Journal of neuroscience: the official journal of the Society for Neuroscience 36(3):837-850. doi:10.1523/jneurosci.4047-15.2016

24. Kissiwaa SA, Patel SD, Winters BL, Bagley EE (2019) Opioids differentially modulate two synapses important for pain processing in the amygdala. Br J Pharmacol. doi:10.1111/bph.14877

25. Kotzer AM (2000) Factors predicting postoperative pain in children and adolescents following spine fusion. Issues Compr Pediatr Nurs 23(2):83-102. doi:10.1080/01460860050121411

26. Lee HJ, Yeomans DC (2014) Opioid induced hyperalgesia in anesthetic settings. Korean journal of anesthesiology 67(5):299-304. doi:10.4097/kjae.2014.67.5.299

27. Li J, Xie W, Zhang JM, Baccei ML (2009) Peripheral nerve injury sensitizes neonatal dorsal horn neurons to tumor necrosis factor-alpha. Mol Pain 5:10. doi:10.1186/1744-8069-5-10

28. Li Z, Li C, Yin P, Wang ZJ, Luo F (2016) Inhibition of CaMKIlalpha in the Central Nucleus of Amygdala Attenuates Fentanyl-Induced Hyperalgesia in Rats. J Pharmacol Exp Ther 359(1):82-89. 
doi:10.1124/jpet.116.233817

29. Li Z, Yin P, Chen J, Jin S, Liu J, Luo F (2017a) CaMKIlalpha may modulate fentanyl-induced hyperalgesia via a CeLC-PAG-RVM-spinal cord descending facilitative pain pathway in rats. PLoS ONE 12(5):e0177412. doi:10.1371/journal.pone.0177412

30. Li Z, Yin P, Chen J, Li C, Liu J, Rambojan H, Luo F (2017b) Activation of the Extracellular SignalRegulated Kinase in the Amygdale Modulates Fentanyl-Induced Hypersensitivity in Rats. The journal of pain: official journal of the American Pain Society 18(2):188-199.

doi:10.1016/j.jpain.2016.10.013

31. Mao J (2002) Opioid-induced abnormal pain sensitivity: implications in clinical opioid therapy. Pain 100(3):213-217. doi:10.1016/s0304-3959(02)00422-0

32. Maxwell LG, Kaufmann SC, Bitzer S, Jackson EV Jr, McGready J, Kost-Byerly S, Kozlowski L, Rothman SK, Yaster M (2005) The effects of a small-dose naloxone infusion on opioid-induced side effects and analgesia in children and adolescents treated with intravenous patient-controlled analgesia: a double-blind, prospective, randomized, controlled study. Anesth Analg 100(4):953-958. doi:10.1213/01.ANE.0000148618.17736.3C

33. Mazzitelli M, Neugebauer V (2019) Amygdala group II mGluRs mediate the inhibitory effects of systemic group II mGluR activation on behavior and spinal neurons in a rat model of arthritis pain. Neuropharmacology 158:107706. doi:10.1016/j.neuropharm.2019.107706

34. Mazzitelli M, Palazzo E, Maione S, Neugebauer V (2018) Group II Metabotropic Glutamate Receptors: Role in Pain Mechanisms and Pain Modulation. Front Mol Neurosci 11:383. doi:10.3389/fnmol.2018.00383

35. More L, Gravius A, Pietraszek M, Belozertseva I, Malyshkin A, Shekunova E, Barberi C, Schaefer D, Schmidt WJ, Danysz W (2007) Comparison of the mGluR1 antagonist A-841720 in rat models of pain and cognition. Behav Pharmacol 18(4):273-281. doi:10.1097/FBP.0b013e3281f19c18

36. Nation KM, De Felice M, Hernandez PI, Dodick DW, Neugebauer V, Navratilova E, Porreca F (2018) Lateralized kappa opioid receptor signaling from the amygdala central nucleus promotes stressinduced functional pain. Pain 159(5):919-928. doi:10.1097/j.pain.0000000000001167

37. Navratilova E, Nation K, Remeniuk B, Neugebauer V, Bannister K, Dickenson AH, Porreca F (2020) Selective modulation of tonic aversive qualities of neuropathic pain by morphine in the central nucleus of the amygdala requires endogenous opioid signaling in the anterior cingulate cortex. Pain 161(3):609-618. doi:10.1097/j.pain.0000000000001748

38. Neugebauer V, Li W, Bird GC, Bhave G, Gereau RWt (2003) Synaptic plasticity in the amygdala in a model of arthritic pain: differential roles of metabotropic glutamate receptors 1 and 5 . The Journal of neuroscience: the official journal of the Society for Neuroscience 23(1):52-63

39. Neugebauer V, Li W, Bird GC, Han JS (2004) The amygdala and persistent pain. The Neuroscientist: a review journal bringing neurobiology. neurology and psychiatry 10(3):221-234.

doi:10.1177/1073858403261077

Page 13/18 
40. Neugebauer V, Mazzitelli M, Cragg B, Ji G, Navratilova E, Porreca F (2020) Amygdala, neuropeptides, and chronic pain-related affective behaviors. Neuropharmacology 170:108052.

doi:10.1016/j.neuropharm.2020.108052

41. Palazzo E, Neugebauer V, Maione S (2019) Editorial: Metabotropic Glutamate Receptors and Neurological/Psychiatric Disorders. Front Mol Neurosci 12:67. doi:10.3389/fnmol.2019.00067

42. Paxinos G, Watson C (2006) The rat brain in stereotaxic coordinates: hard cover edition. Elsevier

43. Ren W, Neugebauer V (2010) Pain-related increase of excitatory transmission and decrease of inhibitory transmission in the central nucleus of the amygdala are mediated by mGluR1. Mol Pain 6:93. doi:10.1186/1744-8069-6-93

44. Ren W, Palazzo E, Maione S, Neugebauer V (2011) Differential effects of mGluR7 and mGluR8 activation on pain-related synaptic activity in the amygdala. Neuropharmacology 61(8):1334-1344. doi:10.1016/j.neuropharm.2011.08.006

45. Roeckel LA, Le Coz GM, Gaveriaux-Ruff C, Simonin F (2016) Opioid-induced hyperalgesia: Cellular and molecular mechanisms. Neuroscience 338:160-182. doi:10.1016/j.neuroscience.2016.06.029

46. Sadler KE, Gartland NM, Cavanaugh JE, Kolber BJ (2017) Central amygdala activation of extracellular signal-regulated kinase 1 and age-dependent changes in inflammatory pain sensitivity in mice. Neurobiol Aging 56:100-107. doi:10.1016/j.neurobiolaging.2017.04.010

47. Sanchez-Blazquez P, Rodriguez-Munoz M, Berrocoso E, Garzon J (2013) The plasticity of the association between mu-opioid receptor and glutamate ionotropic receptor $\mathrm{N}$ in opioid analgesic tolerance and neuropathic pain. Eur J Pharmacol 716(1-3):94-105.

doi:10.1016/j.ejphar.2013.01.066

48. Shin S, Kwon O, Kang Jl, Kwon S, Oh S, Choi J, Kim CH, Kim DG (2015) mGluR5 in the nucleus accumbens is critical for promoting resilience to chronic stress. Nat Neurosci 18(7):1017-1024

49. Terashvili M, Wu HE, Schwasinger E, Tseng LF (2007) Paradoxical hyperalgesia induced by muopioid receptor agonist endomorphin-2, but not endomorphin-1, microinjected into the centromedial amygdala of the rat. Eur J Pharmacol 554(2-3):137-144. doi:10.1016/j.ejphar.2006.10.014

50. Thompson JM, Neugebauer V (2017) Amygdala Plasticity and Pain. Pain research \& management 2017:8296501. doi:10.1155/2017/8296501

51. Tripi PA, Kuestner ME, Poe-Kochert CS, Rubin K, Son-Hing JP, Thompson GH, Tobias JD (2015) Intrathecal morphine attenuates acute opioid tolerance secondary to remifentanil infusions during spinal surgery in adolescents. Journal of pain research 8:637-640. doi:10.2147/JPR.S88687

52. Tully K, Li Y, Bolshakov VY (2007) Keeping in check painful synapses in central amygdala. Neuron 56(5):757-759. doi:10.1016/j.neuron.2007.11.011

53. Vanderah TW, Suenaga NM, Ossipov MH, Malan TP Jr, Lai J, Porreca F (2001) Tonic descending facilitation from the rostral ventromedial medulla mediates opioid-induced abnormal pain and antinociceptive tolerance. The Journal of neuroscience: the official journal of the Society for Neuroscience 21(1):279-286 
54. Wasserman RA, Hassett AL, Harte SE, Goesling J, Malinoff HL, Berland DW, Zollars J,Moser SE, Brummett CM (2015) Pressure Pain Sensitivity in Patients With Suspected Opioid-Induced Hyperalgesia. Regional Anesthesia \& Pain Medicine 40:687-693

55. Xu H, Wu LJ, Wang H, Zhang X, Vadakkan KI, Kim SS, Steenland HW, Zhuo M (2008) Presynaptic and postsynaptic amplifications of neuropathic pain in the anterior cingulate cortex. The Journal of neuroscience: the official journal of the Society for Neuroscience 28(29):7445-7453. doi:10.1523/JNEUROSCI.1812-08.2008

56. Zapala MA, Hovatta I, Ellison JA, Wodicka L, Del Rio JA, Tennant R, Tynan W, Broide RS, Helton R, Stoveken BS, Winrow C, Lockhart DJ, Reilly JF, Young WG, Bloom FE, Lockhart DJ, Barlow C (2005) Adult mouse brain gene expression patterns bear an embryologic imprint. Proc Natl Acad Sci USA 102(29):10357-10362. doi:10.1073/pnas.0503357102

57. Zimmermann M (1983) Ethical guidelines for investigations of experimental pain in conscious animals. Pain 16(2):109-110. doi:10.1016/0304-3959(83)90201-4

\section{Figures}

\section{Figure 1}

Fentanyl injection induces mGluR1 up-expression in the right CeLC. (A) Flow diagram of experimental design. Western blot was performed $6 \mathrm{~h}$ after fentanyl injection. (B) Representative immunoblots probed with mGluR1 after stimulation with fentanyl or saline. 6 CelCs were collected per sample. (C) Immunohistochemistry analysis of the right CeLC using an anti-mGluR1 antibody counter stained with hematoxylin. The mGluR1 expression is marked by an arrow. Scale bar denotes $20 \mu \mathrm{m}$. Rat Brain Atlas is from http://labs.gaidi.ca/rat-brain-atlas/ (Paxinos and Watson 2006).

\section{Figure 2}

The CeLC glutamate content is significantly increased in the case of fentanyl treatment and is decreased by A841720 microinjection. (A) Flow diagram of the experimental design. Rats were subcutaneously injected with fentanyl 7 days after CeLC cannulas, and received A841720 at $6 \mathrm{~h}$ after the fentanyl treatment. Rat glutamic acid ELISA analysis was performed 30 min later. (B) Cannula location. CeLC was histologically verified with eosin. Rat Brain Atlas is from http://labs.gaidi.ca/rat-brain-atlas/(Paxinos and Watson 2006). (C) Representative schematic illustration of the location for A841720 application into the CeLC. (D) The CeLC glutamate content measured by ELISA. Data was presented as mean \pm SD. Each sample was collected from 7 rats. Number means biological replicates, $n=(7,6,6,6)$. One-way ANOVA followed by Tukey Post hoc, $F(3,21)=40.03, P<0.0001 .{ }^{*} p<0.05,{ }^{*} p<0.01, * \star * p<0.001$, exact $p$-value 
were marked if it $>0.001$. Total sample size $=8$. The data are representatives of three independent experiments.

\section{Figure 3}

The increase in mEPSC frequency and amplitude in OlH rats is reversed by administration of A841720 to brain slices. (A) Flow diagram of the experimental design. The CeLC slices were prepared at $6 \mathrm{~h}$ after the last fentanyl dose. The A841720 solution was introduced to the recording well 10 min after the baseline recording. (B) Representative mEPSC recordings from CeLC neurons. Original current traces of mEPSC were recorded in an individual CeLC neuron treated by fentanyl in the absence or presence of $\mathrm{A} 841720$. The frequency (C) and amplitude (D) of mEPSCs were considerably higher in the fentanyl group compared with the saline group, and then returned to normal levels after the application of A841720. Data was presented as mean \pm SD. One-way ANOVA followed by Tukey Post hoc. $n=(6,6,6,5), F(3,19)=$ 9.799, $P=0.0004(C), n=(6,6,5,5), F(3,18)=12.91, P<0.0001$ (D). $n s p>0.05,{ }^{*} p<0.01, \star \star \star x p<0.001$, exact $p$-value were marked if it $>0.001$. Total sample size $=12(C, D)$. Number means biological replicates. The data are representatives of three independent experiments. (E) Cumulative probability of the interevent interval of mEPSCs in right CeLC. Kolmogorov-Smirnov test. Saline + Vehicle: Saline + A841720, ns $p>0.05$; Saline + Vehicle: Fentanyl + Vehicle, $p<0.0001$; Fentanyl + Vehicle: Fentanyl + A841720, $p<$ 0.0001. (F) Cumulative probability of mEPSC amplitude in CeLC. Kolmogorov-Smirnov test. Saline + Vehicle: Saline + A841720, ns p >0.05; Saline + Vehicle: Fentanyl + Vehicle, $p<0.0001 ;$ Fentanyl + Vehicle: Fentanyl + A841720, $p<0.0001$. The data are representatives of three independent experiments. 
A

Time after Fentanyl

\begin{tabular}{lc|lcc|}
\hline D.7 & D.1 & Do/Oh & $6 h$ & $6.5 \mathrm{~h}$ \\
\hline $\begin{array}{l}\text { Nociceptive tests } \\
\text { CeLC Cannulation }\end{array}$ & Nociceptive tests & $\begin{array}{l}\text { Fentanyl } \\
\text { or Saline }\end{array}$ & $\begin{array}{l}\text { A841720 Nociceptive tests } \\
\text { or Vehicle }\end{array}$
\end{tabular}

B

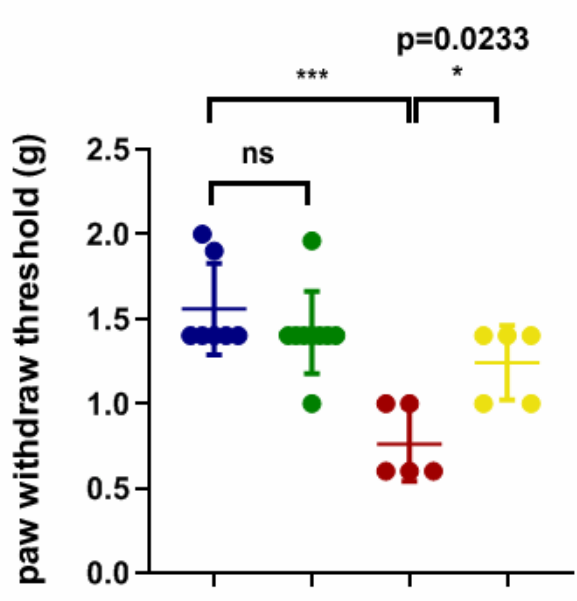

C
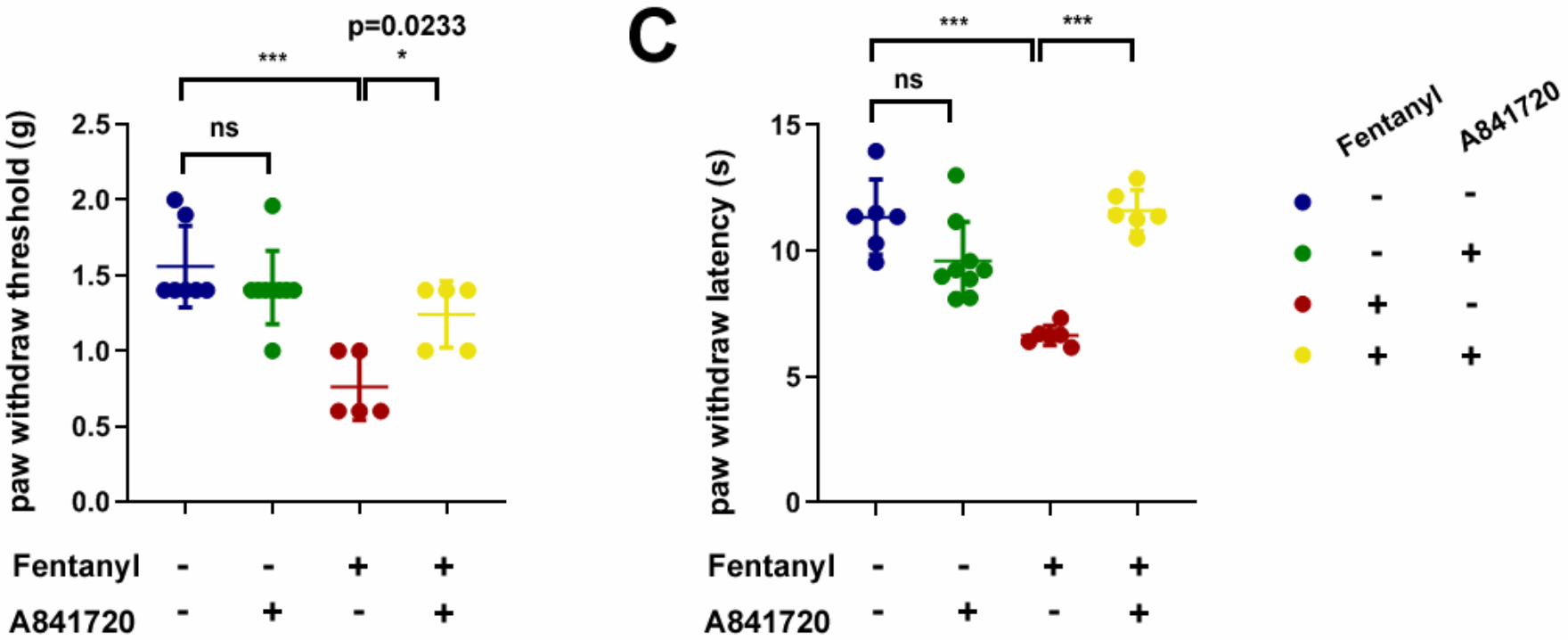

$\begin{array}{lllll}\text { Fentanyl } & - & - & + & + \\ \text { A841720 } & - & + & - & +\end{array}$

Figure 4

Microinjection of A841720 into CeLC blocks hyperalgesia induced by fentanyl. (A) Flow diagram of the experimental design. Rats were subcutaneously injected with fentanyl 7 days after CeLC cannulas, and received respectively A841720 at $6 \mathrm{~h}$ after the fentanyl treatment. Nociceptive tests were performed 30 min later. The paw withdraw threshold (B) and the paw withdraw latency (C) were determined 30 min after A841720 microinjection ( $20 \mathrm{nmol} / \mathrm{mL})$ into CeLC. Data was presented as mean \pm SD. One-way ANOVA followed by Tukey Post hoc, $n=(7,9,5,5), F(3,22)=11.71, P<0.0001(B), n=(6,9,6,6), F(3,23)$ $=20.55, P<0.0001(C) .{ }^{*} p<0.05,{ }^{* *} p<0.01,{ }^{* *} p<0.001$, exact $p$-value were marked if it $>0.001$. Total sample size $=12(B, C)$. Number means biological replicates. The data are representatives of three independent experiments.

\section{Supplementary Files}

This is a list of supplementary files associated with this preprint. Click to download.

- abstract.tif

- FigS.docx

- s1.tif 
- s2.tif

Page 18/18 